\title{
External audit of providers of the Cervical Cancer Prevention Programme in Poland in 2016/2017
}

\author{
Maryla Turkot ${ }^{1}$, Dagmara Mokwa ${ }^{1}$, Paulina Wieszczy ${ }^{1,2}$, Katarzyna Spych ${ }^{1}$, \\ Natalia Kurowska ${ }^{1}$, Andrzej Nowakowski 1, 3, 4 , Michał F. Kamiński 1, 2, 5, 6
}

Introduction. The Cervical Cancer (CC) Prevention Programme includes 3 phases: basic (Pap smear collection), diagnostic (Pap smear evaluation) and in-depth (colposcopy/biopsy in case of abnormal smear test findings). The Programme service providers are subject to an external audit and this publication's objective is to analyse its results from 2016 and the first half of 2017.

Materials and methods. The audit of the Programme performance in the period 01.01.2016-30.06.2017 was carried out by external auditors by way of personal visits to the offices of the service providers and by way of direct data retrieval. The audit covered $12 \%$ (198) of the basic phase, $100 \%(66)$ of the diagnostic phase and $100 \%(62)$ of the in-depth phase facilities. The Polish National Health Fund (NHF) did not make available the routinely collected data for the purpose of audit. Audit data collected in the developed protocols were analysed.

Results. The number of Pap smears $(2,028,988)$ and the number of colposcopies $(13,636)$ outside the Programme was, respectively, more than two and three times higher than in the Programme (cytology — 801,640, colposcopy - 3929). The performance of the procedures (Programme vs outside the Programme) was highly variable depending on the provider. The percentage of Pap smears unsuitable for evaluation did not differ significantly between gynaecologists and midwives. All audited cytological laboratories carried out rescreening of samples. Biopsy was not performed in $11 \%$ (2016) and 15\% (2017) of colposcopy laboratories. Inaccuracies were found in 19\% (61) of the audit protocols.

Discussion. Significantly higher number of procedures performed outside the Programme results from lower renumeration of procedures within the Programme. Variable provider's preferences in the mode of procedures execution indicates that with the use of appropriate organisational solutions it would be possible to reduce opportunistic screening, which is of unknown quality. The quality of Pap smear sample collection in the case of gynaecologists and midwives is the same, but the number of primary care provider (PCP) facilities where midwives collect smears is very limited. The inaccuracies noted in the audit protocols indicate that the lack of access to data collected by the National Health Fund decreased the quality of the audit carried out and the reliability of the data obtained.

Conclusions. Restoring full access to data collected by the NHF is crucial for the Programme audit quality. Measures should be implemented to reduce opportunistic screening and shift the stream of tests to the Programme (both at the basic and at the in-depth phase), and to increase the availability of tests in PCP facilities through training for midwives.

NOWOTWORY J Oncol 2018; 68, 2: 65-78

Key words: cervical cancer screening, cytology, cervical cancer prevention

\footnotetext{
${ }^{1}$ Department of Cancer Prevention, Maria Skłodowska-Curie Memorial Cancer Center and Institute of Oncology, Warszawa, Poland

${ }^{2}$ Department of Gastroenterology, Hepatology and Oncology, Medical Centre for Postgraduate Education, Warszawa, Poland ${ }^{3}$ Second Department of Gynaecological Oncology, St. John's Cancer Centre of Lublin, Poland

${ }^{4}$ Institute of Health Sciences, University of Life Sciences and Humanities, Siedlce, Poland

${ }^{5}$ Department of Gastroenterological Oncology, Maria Skłodowska-Curie Memorial Cancer Center and Institute of Oncology, Warszawa, Poland

${ }^{6}$ Department of Health Management and Health Economics, University of Oslo, Norway
} 


\section{Introduction}

The objective of the Cervical Cancer Prevention Programme in Poland, implemented in 2006, is to further reduce the incidence and mortality of cervical cancer in women [1].

Under the Programme, each insured Polish woman aged 25 to 59 is entitled to a free of charge Pap smear test if she has not received it within the Programme in the last 3 years.

The service providers perform the Programme in 3 phases:

1) basic phase: collection of cervical material for cytological screening in the framework of:

a) outpatient specialist care in the field of obstetrics and gynaecology, as consultation from the catalogue of separate specialised services;

b) primary health care provided by PCP midwives;

2) diagnostic phase: performing microscopic evaluation of cytological material sent by the diagnostic phase provider of the Programme and reporting in the computer system;

3) in-depth diagnostic phase: colposcopy or colposcopy with targeted biopsy and histopathological examination in women with abnormal results of Pap smear test obtained within the Programme.

Service providers of each phase must meet the requirements set out in the regulation of the Minister of Health, Journal of Laws of 2016 pos. 1372, Journal of Laws of 2016 pos. 1743 and the order of the President of the National Health Fund (NHF) no. 81/2013/DSOZ concerning the detailed criteria for selection of healthcare service providers such as healthcare programs.

The tests carried out under the Programme are unlimited and financed by the NHF. Data concerning all medical services provided under the Programme are collected in the Information System for Prevention Monitoring (SIMP), which is an electronic database accessible for every healthcare provider.

In 2017, there were 1663 service providers performed the basic phase, of which 1602 were outpatient specialist care institutions in the field of obstetrics and gynaecology and 61 PCP facilities, 66 facilities contracted for the performance of the diagnostic phase, and 62 providers contracted for the performance in-depth diagnostic phase.

In 2017, the coordination of the Programme in Poland was carried out by the Central Coordination Centre (COK Centralny Ośrodek Koordynujący) within the Department of Cancer Prevention,The Maria Sklodowska-Curie Institute Oncology Center, Warsaw, Poland, based on a contract with the Ministry of Health. The tasks of COK included, among others, quality control of services provided at each phase of the Programme in 2016 and the first half of 2017.

According to the European Guidelines for Quality Assurance in Cervical Cancer Screening [2], quality control of cytological screening is one of the key elements for ensuring the effectiveness of the Programme. In accordance with the Guidelines, the results of the quality control should be published periodically. The objective of this publication is to analyse the results of external audit of the providers of the Cervical Cancer Prevention Programme in Poland (as one of the elements of the Programme quality control) in 2016 and the first half of 2017.

The specific objectives were:

- Evaluation of the preparation of facilities (e.g. equipment, organisation of the facility, qualifications of employees, procedures in place) for the implementation of tasks resulting from the Programme.

- Comparison of the number of tests performed under the organised Programme to the number of tests performed under opportunistic screening.

- Evaluation of the quality of the smears taken depending on the profession (gynaecologist or midwife).

- Analysing follow-up of patients with positive Pap smear findings.

- Evaluation of the current method of conducting audits of facilities implementing the Cervical Cancer Prevention Programme.

The research theses were:

- The majority of facilities implementing the Programme at the basic, diagnostic and in-depth stages are prepared in terms of equipment, organisation of the facility, qualifications of their employees, procedures in place for the performance of tasks resulting from the contract.

- Both at the basic and in-depth stages, the test performed in patients outside the Prevention Programme outnumber the tests within the Programme.

- The quality of Pap smears taken by midwives and gynaecologists, measured as a percentage of the results unsuitable for evaluation, is statistically the same.

- Some patients with positive results of cytological test in the Programme perform further diagnostics beyond the in-depth stage of the Programme or do not perform it at all.

- The current way which the facilities implementing the Cervical Cancer Prevention Programme are audited, dictated by the lack of access to data collected in SIMP for COK employees and auditors is insufficient and requires fundamental changes.

\section{Material and methods}

Due to the lack of access to the SIMP for COK employees by the National Health Fund, a decision was made to carry out audits by way of personal visits of inspectors to the offices of the service providers and to obtain data directly.

The contractor for the audit was selected through an open tender procedure. The audit was carried out by 14 auditors from 12 October to 28 November, 2017. 
The audit covered $12 \%$ (198) of the basic phase facilities, selected at random, $100 \%$ (66) of the diagnostic phase and $100 \%$ (62) of the in-depth phase.

The auditors collected data in audit reports based on the documentation of the service providers, data obtained from SIMP at the service provider level and the electronic systems of service providers. The adoption of this mode of data collection was caused by the lack of access to SIMP from the level of COK and the auditors themselves.

At various stages, the scope of the audit included analysis of, among other things:

1. Basic phase

- Working hours and the test result waiting time

- Qualifications of the staff employed

- Equipment of the lab rooms and the equipment used in Pap smear tests

- Methods of marking the slides and archiving the results

- Results of collected cervical screening samples

2. Diagnostic phase

- Number of Pap smears assessed within and outside the Programme

- Results of Pap smear tests within the Programme

- The conditions of providing services guaranteed as per the regulations of the Minister of Health, Journal of Rights no. 2016 pos. 1372, and Journal of Rights no. 2016 pos. 1743 , satisfied by the personnel and the laboratory

3. In-depth diagnostics phase

- Facility's work organisation

- Number of targeted biopsies performed within the Programme

- Equipment in the lab rooms.

\section{Statistical methods}

Continuous variables were described using mean, median and range (minimum, maximum). Contingency tables were used to describe categorised variables. The linear relationship between continuous variables was investigated using Spearman's rank correlation coefficient. All tests were performed at statistical significance of 0.05. Data analysis was conducted with the Stata statistical package, ver. 13.1 (Stata Corporation, College Station, Texas, USA).

\section{Results}

\section{Basic phase}

185 out of 1602 facilities implementing the Programme under a contract for outpatient specialist care in the field of obstetrics and gynaecology and 15 out of 61 facilities implementing the Program under the Primary Health Care were selected for audit. In total, the audit covered 198 units implementing the Programme (Fig. 1).

Table I (Appendix) provides basic data on the working time of the facilities audited, the number of gynaecologists and mid wives employed and the waiting time declared and confirmed by the auditors for Pap smears collection, as well as the number of Pap smears collected.

The total number of Pap smear tests taken in the audited facilities outside the Programme was nearly twice as high as the number of Pap smears taken within the Programme in the analysed period (Fig. 2). The ratio of the number of Pap smears taken in particular facilities within the Programme to those taken outside the Programme varied greatly and ranged from 0.0 to 7.4 .

There was a weak correlation between the number of working days per week and the declared and confirmed of waiting days for the collection of Pap smear (Spearman's rank correlation coefficient $-0.201(p=0.005)$ and -0.164 $(p=0.021)$, respectively). There was no correlation between the number of Pap smears taken in both periods and the number of days in waiting for the collection of a Pap smear at the facility verified by the auditors (Spearman's rank correlation coefficient $=0.079(p=0.271))$.

The waiting time for the result of the cervical screening test declared by the basic phase facilities was less than 15 days in 146 clinics (73.7\% of those audited). In the remaining $52(26.3 \%)$ it was longer than 15 days.

Table II (Appendix) provides basic data on the percentage of Pap smears classified as "unsuitable for evaluation". In general, this percentage was very low except for several facilities. No statistically significant difference was found in

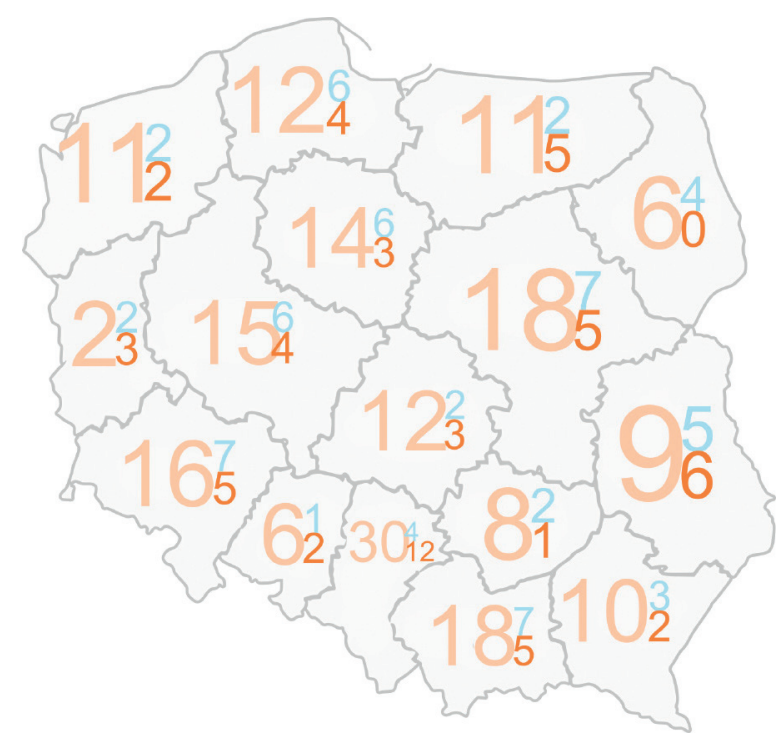

Figure 1. Number of facilities audited in 2017 by voivodeships - The number of facilities in each voivodeship carrying out the basic phase amounts to approx. $12 \%$

The number of facilities in the voivodeship carrying out the diagnostic phase amounts to $100 \%$

- The number of facilities in the voivodeship carrying out the phase of in-depth diagnostics amounts to $100 \%$ 


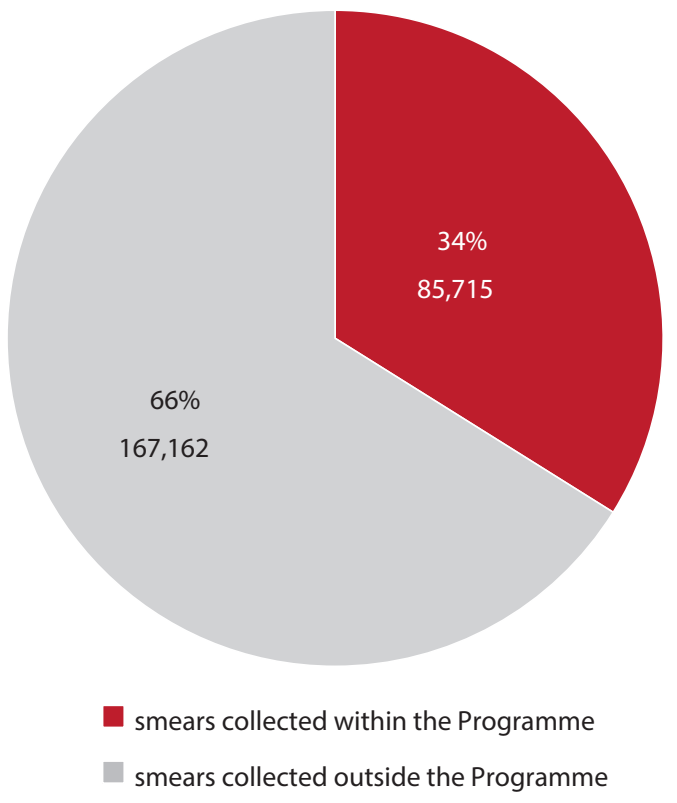

Figure 2. Number of Pap smear samples taken at the basic phase facilities audited

the percentage of Pap smears collected by gynaecologists and midwives classified as "unsuitable for evaluation" (Fig. 3). These results apply to all the test samples collected at the facilities (within and outside the Programme). The percentage of Pap smears unsuitable for evaluation within the Programme was lower and amounted to $0.62 \%$.

A weak correlation was found between the percentage of Pap smears classified as "unsuitable for evaluation" and

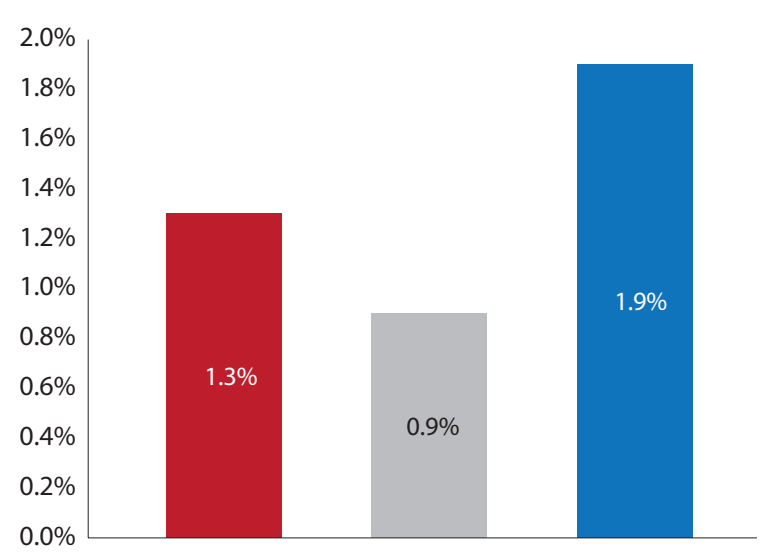

Average percentage

Percentage of smears unsuitable for evaluation in audited facilities

Percentage of smears unsuitable for evaluation collected by gynaecologists in audited facilities

Percentage of smears unsuitable for evaluation collected by midwives in audited facilities

Figure 3. Average percentage of smears unsuitable for evaluation in the primary phase facilities audited - all tests (within the Programme and outside the Programme) the total number of test samples collected by gynaecologists (Spearman's rank correlation coefficient $=0.136$ $(p=0.003)$ ). No such correlation was observed among midwives (Spearman's rank correlation coefficient $=0.117$ $(p=0.234))$.

In both audited periods the percentage of positive results did not exceed $3 \%$.

The follow-up of patients with positive cervical screening test results obtained at audited basic phase facilities were presented in Table III (Appendix).

The percentage of women in whom, in accordance with the criteria adopted in the Programme, further diagnosis was performed at the in-depth phase or the Pap smear was repeated within the Programme was $47.92 \%$ in all audited periods. About $8.5 \%$ of patients with positive results were admitted to further diagnostics under procedures reimbursed by the NHF outside the Program. Service providers have data on follow-up of patients or have contacted another $16.72 \%$ of patients with positive results of cervical screening test for whom data were not available in the SIMP and in the NHF reporting data on further diagnosis. There are no data on follow-up of $5.98 \%$ of patients with positive results of cervical screening test performed within the Programme (Fig. 4). Due to the inaccuracies in the post-audit protocols, the total number of patients for whom further fates were described does not equal the number of all patients with positive results of cervical screening test. During the analysis of the results, 22 (11\%) erroneously filled in protocols were found.

The basic data on the equipment in facilities are presented in Table IV (Appendix). Almost all facilities were equipped with elements necessary for the proper collection of cytological smears, i.e. a gynaecological chair, specula, disposable brushes, microscope slides and a fixative.

\section{Diagnostic phase}

The audit covered 66 facilities implementing this diagnostic stage. The largest number of cytological laboratories implementing this phase is located in Dolnośląskie, Małopolskie and Mazowieckie Voivodeships (each with 7 facilities). The smallest number is in the Opolskie Voivodeship, one lab only (Fig. 1). During the analysis of the results, inaccuracies were found in 30 (45.5\%) audit protocols.

Figure 5 presents basic data on the number of cervical screening tests performed in the audited laboratories. Details, including the breakdown by year, average, median, minimum and maximum values, are given in Table V (Appendix).

In 2016, 14,589 positive result sand 3196 unsuitable for evaluation smears were found, representing $2.69 \%$ and $0.56 \%$ respectively of the total number of tests evaluated in the Programme. In the first half of 2017, 6500 positive results and 1536 smears unsuitable for evaluation were found, representing $2.61 \%$ and $0.65 \%$ respectively of the 


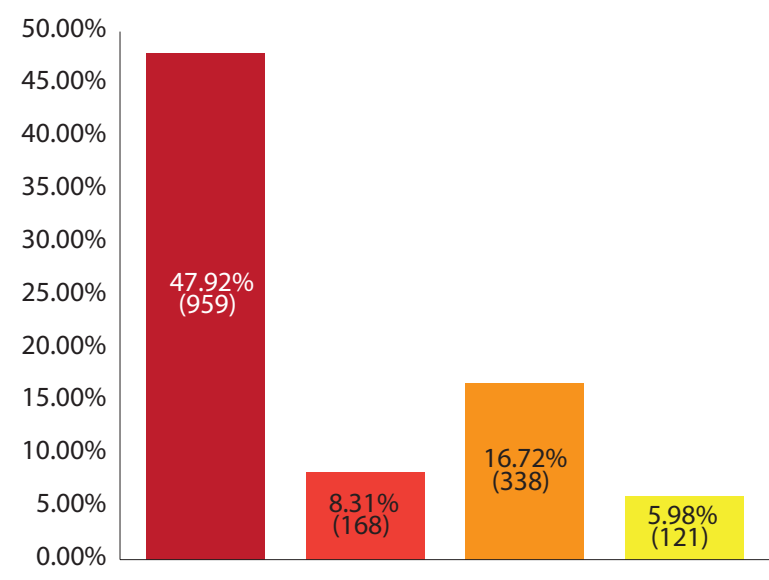

Percentage of all positive results in the Programme* (in brackets: the number of women)

* Due to the inaccuracies in the post-audit protocols, the total number of patients for whom further follow-up was described does not equal the number of all patients with positive results of cervical screening test.

Women at whom further diagnostics was performed at in-depth phase or the Pap smear test was repeated as per SIMP recommendation

Women at whom neither further diagnostics was performed at in-depth phase nor the Pap smear test was repeated as per SIMP recommendation, but there are NHF report data available

Women for whom there is no in-depth phase/repeated test data available in SIMP and reporting data are not available in SIMP, but there are data on follow-up/contacts with the patient in the facility documentation

Women for whom there is no in-depth phase/repeated test data available in SIMP and reporting data are not available in SIMP, and there are no data on follow-up/contacts with the patient in the facility documentation

Figure 4. Patients with positive results of cervical screening tests follow-up

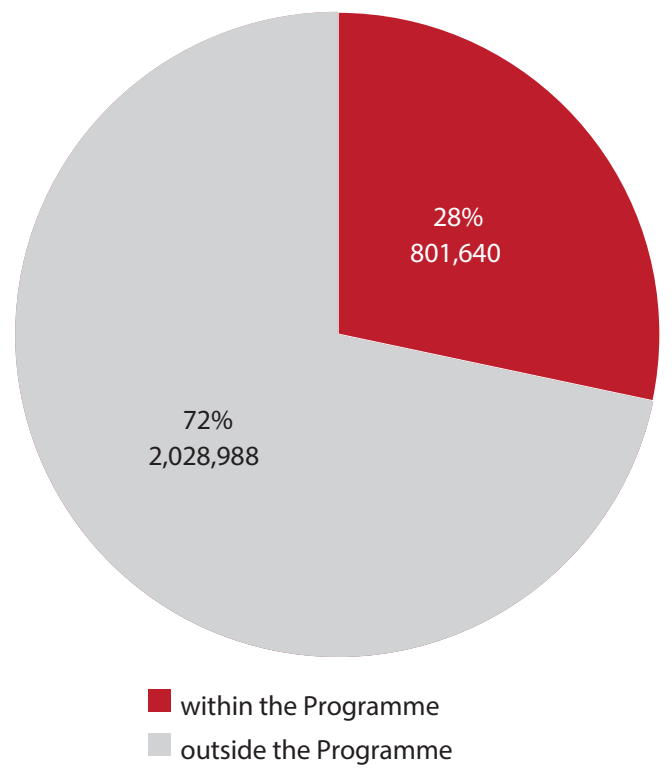

Figure 5. Number of Pap tests taken at the diagnostic phase facilities audited total number of tests evaluated in the Programme in the analysed period (Fig. 6).

Table VI contains numbers and percentages of positive cervical screening results found in the audited laboratories, while tables VII and VIII contain numbers and percentages of particular categories of abnormal cervical screening results found in the Bethesda system in the audited laboratories during the two analysed periods (all tables are enclosed).

As part of internal quality control, cytological rescreening is carried out by most cytology laboratories, but in different forms (Fig. 7). 86.4\% of the laboratories perform a full re-evaluation of $10 \%$ of the evaluated cytology samples, $24.2 \%$ of the laboratories perform a full re-evaluation of all samples, and $28.8 \%$ of the laboratories perform the re-evaluation in a different way. On average, the re-evaluation

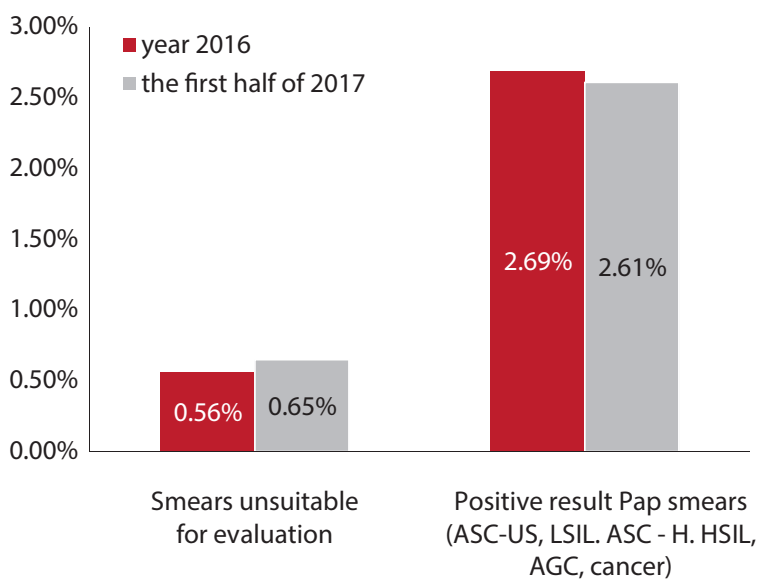

Figure 6. Percentage of test results, positive and unsuitable for evaluation in the audited facilities at the diagnostic phase (in relation to all tests within the Programme)

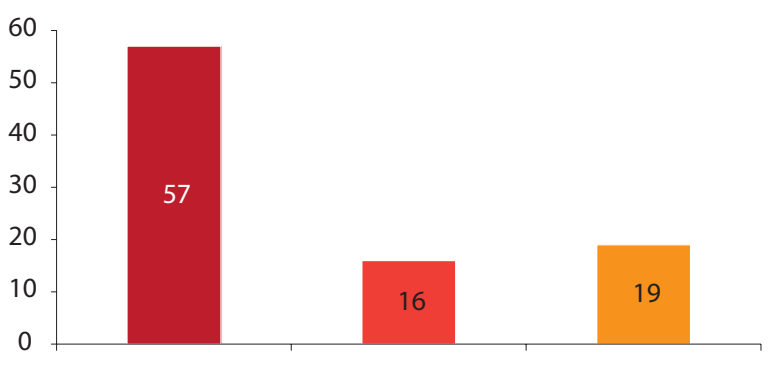

laboratories performing full rescreening of $10 \%$ of evaluated Pap smear samples

laboratories performing full rescreening of all of evaluated Pap smear samples

laboratories performing other method of rescreening

Figure 7. Laboratories performing rescreening of cytology samples - diagnostic phase 
concerned $23.1 \%$ and $25.1 \%$ of samples in 2016 and the first half of 2017, respectively. Basic data on the percentage of the samples re-evaluated and on the discrepancies found between the initial and the re-evaluation are given in Table IX (Appendix).

Staining with Papanicolaou technique is performed by $66(100 \%)$ laboratories, the same number has "dotted" samples with positive results. One laboratory did not have Papanicolaou staining reagents, although all of them declared staining with the method mentioned above. Only $41(65.1 \%)$ laboratories keep electronic documentation, 16 (25.4\%) - both electronic and paper one, and 6 (9.5\%) uses only paper one. There are no data for 3 laboratories in this respect. The continuity of numbering is ensured by $63(95.5 \%)$ laboratories, while in 3 (4.5\%) the continuity of numbering of preparations is not kept. All audited laboratories are equipped with high quality microscopes. Correct archiving of samples in accordance with the regulation of the Minister of Health of 21 January 2009 amending the regulation on quality standards for medical diagnostic and microbiological laboratories and guidelines of the Polish Society of Pathologists (PSP Licence Commission) is carried out in 66 (100\%) laboratories. Finding archived samples in an easy way can be done in 66 (100\%) facilities. The minimum, maximum, average and median time of finding an archived sample in the facilities is, respectively: $0.05 \mathrm{~h}, 48 \mathrm{~h}$, $1.6 \mathrm{~h}$ and $0.32 \mathrm{~h}$.

The general evaluation of the audit of cytological laboratories implementing the diagnostic phase of the Cervical Cancer Prevention Programme and the data concerning the fulfilment of particular conditions of the Programme implementation are presented in Table X (Appendix). 40 (60.6\%) of laboratories received recommendations after the audit.

\section{In-depth phase}

The audit of the in-depth diagnostics stage of the Cervical Cancer Prevention Programme implemented in Poland was carried out for the second time since the beginning of the Programme. The audit covered 62 units implementing indepth diagnostic phase. The largest number of laboratories implementing this phase is located in Śląskie Voivodeship (12 laboratories), whereas in Podlaskie Voivodeship there is not a single laboratory (Fig. 1). During the analysis of the results, inaccuracies were found in $14.5 \%$ post-audit protocols.

Table XI (Appendix) provides basic data on the working time of the facilities audited, the number of gynaecologists and midwives employed and the time of waiting for colposcopy declared and confirmed by the auditors. The declared and confirmed times of waiting for colposcopy were very similar.

The number of colposcopies performed is shown in Table XII (Appendix). It shows that the number of colposcopies performed outside the Programme is almost four times higher than the number of tests performed within the Programme (Fig. 8). The percentage of cervical biopsies performed in relation to colposcopies is presented in Table XIII (Appendix).

Data on the equipment and tools necessary to collect tissue samples for histopathological examination, available in the laboratories, are presented in Table XIV (Appendix). 3 $(4,8 \%)$ labs do not have biopsy forceps, while $4(6,4 \%)$ labs do not have cervical scrapers and Lugol's iodine. 10 (16.1\%) laboratories did not have a sterilisation procedure in place, and at the same time 6 (9.7\%) declared to use only single-use instruments. The minimum, maximum, mean and median times of waiting for the histopathological examination were respectively: $3.3,28.9,11.8$ and 11.6 days.

\section{Discussion}

Ensuring high quality procedures at each phase of organised cervical cancer screening is one of the key factors (apart from wide coverage of the target population and high quality treatment of detected pre-cancerous conditions) determining the effectiveness of secondary prevention [2].

Audits of the Programme service providers commissioned by the COK were one of the elements of evaluation and attempts to ensure high quality of services offered under the Programme. Unfortunately, the complete lack of access to data collected at SIMP due to procedural reasons (between the ministry of Health and the National Health Fund) made the audit more complicated to a significant degree and certainly reduced the quality of audit and the reliability

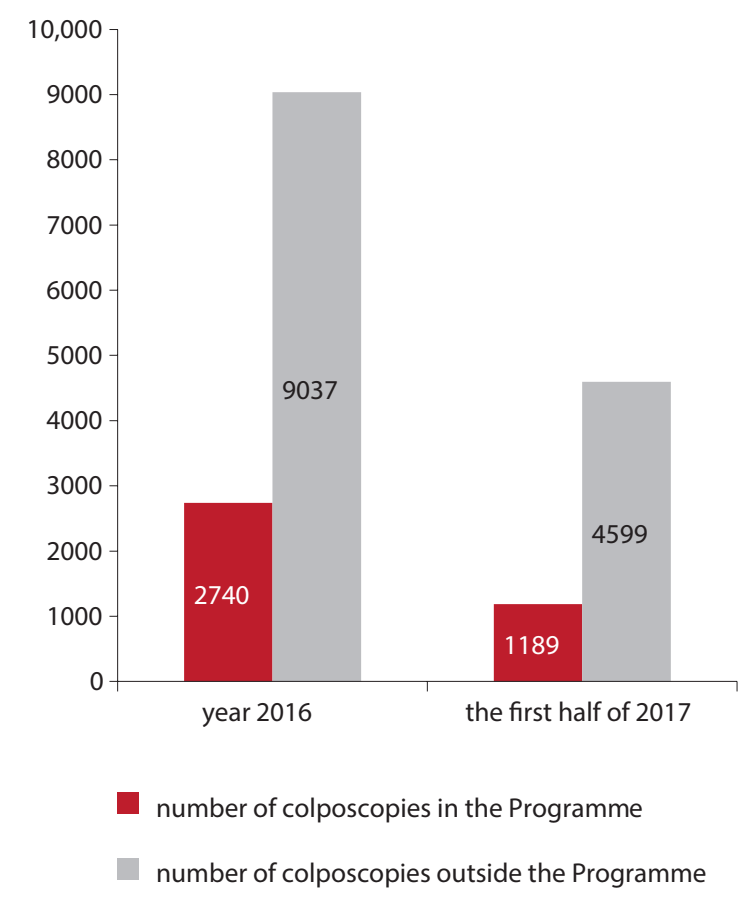

Figure 8. Number of colposcopies performed within and outside the Programme 
of the data obtained. The post-audit reports provided by the auditors contained inaccuracies. Restoring access to SIMP data for COK staff is absolutely crucial to continue the quality control of services in the Cervical Cancer Prevention Programme. This will allow for avoiding the need for the auditors' obtaining data (collected in SIMP but not made available) directly from the service providers. Moreover, dedicated COK staff should have access to individual records and not only to aggregated data available in SIMP reports. Properly secured access to the required data should enable broad evaluation of the quality of procedures in the Programme, based, among others, on the calculation of quality indicators and measures recommended by the EU Guidelines [2].

The data from the audit of all 3 stages of the Programme clearly indicate that the number of Pap tests and colposcopies performed outside the Programme is much higher than the number of tests performed within the Programme in Poland. This is confirmed by the fact that opportunistic screening, less cost-efficient, is more common than organised screening, which has been observed since the beginning of the Programme implementation. Additionally, it should be emphasized that the number of cytological laboratories participating in the Programme is only a certain percentage (due to the lack of a central register) of all cytological laboratories operating in Poland, and the total number of tests conducted is unknown, but may reach several million annually. The number of colposcopies performed outside the Programme under the AOS is four times higher than in the Programme (only in the clinics performing the in-depth phase), and it is certainly a result of the lower pricing of procedures under the Programme in relation to the AOS. Significant differentiation of service providers' preferences within the implementation of preventive procedures (Programme vs AOS) indicates that by applying appropriate organisational solutions (appropriate pricing of Programme procedures, limiting access/benefit indications outside the Programme), it is possible to limit ineffective opportunistic screening. At the same time, it should be noted that PCP facilities are only authorised to carry out procedures under the Programme and do not carry out opportunistic screening financed by the National Health Fund. Moreover, in small PCP facilities with a limited number of patients aged 25-59, the purchase of a gynaecological chair for the implementation of the Programme may not be cost-effective as PCP facilities may implement the Programme only within the scope of the list of their patients.

The lack of correlation between the number of collected Pap smears and the number of waiting days verified by the auditors may indicate that the number of days waiting for the collection of a Pap smear depends on the organisation of the facility and not on the demand for tests. This aspect requires further analysis.
A weak but statistically significant relationship between the percentage of "unsuitable for evaluation" smears and the total number of samples taken in facilities requires further analysis and attention to be paid to the quality of the collection of Pap smears in large facilities.

The percentage of smears classified as "unsuitable for evaluation" is low. Since no differences were found in the percentage of cervical smears collected by gynaecologists and midwives assessed as "unsuitable for evaluation", it is justified to support the collection of samples by midwives, especially in PCP facilities, where only screening within the Programme is performed. Further training by the COK and an increase in the number of midwives taking samples should increase the availability of cervical screening tests in the Programme.

There are significant differences in the percentage of positive cervical screening tests and diagnosed pathological changes in individual laboratories, which indirectly indicates differences in the quality of smear evaluation. This is particularly true for ASC-US and LSIL smears. Currently, the lack of data concerning colposcopy and histological verification of individual positive cervical screening tests and access to data collected in the IT System for Monitoring Prevention precludes the final analysis and drawing conclusions regarding the quality of diagnoses in individual laboratories. As part of internal quality control, cytological rescreening is commonly carried out by most cytology laboratories, but in different forms. On the other hand, external quality control of laboratories' operation and individual cytotechnologists is recommended by the EU Guidelines and should be implemented in Poland.

Considering the difficulties in accessing histopathological examinations, mainly related to a small number of pathomorphologists practising in Poland, the time of waiting for the result of histopathological examination in the Programme should be considered acceptable. The conditions affecting the quality of the tests, such as: the method of staining the samples, marking samples with positive results, continuity of samples numbering, access to high quality microscopes are fulfilled in almost all laboratories implementing the Programme, while the time of access to archived samples is varied and should be shortened in some audited laboratories.

We found large differences in the frequency of histopathological verification performed by colposcopy facilities. This issue requires further analysis, searching for the reasons for such differentiation and examining the potential impact of this situation on the quality of final diagnoses and the impact on the fate of patients with abnormal results of cervical screening tests. There is a need to unify the rules of procedure for histopathological verification of abnormal cervical smears in laboratories offering the in-depth diagnostics phase. The period of waiting for the colposcopy 
was relatively short and should not delay the diagnosis. The analysis shows that the percentage of patients subject to verification of cervical screening tests positive results under the Programme remains insufficient and a significant part of positive test results is still subject to further diagnosis outside the Programme. The percentage of recommendations implementation for in-depth diagnostics in previous years ranged from $29.7 \%$ to $50.5 \%$ [3]. The results of colposcopies and histopathological examinations performed outside the Program are not recorded in SIMP. This prevents the calculation of quality indicators and analysis of cytologic-histologic correlation.

The percentage of patients with cervical smear results, whose fate is unknown, is relatively low, but taking into account the estimated number of positive cervical screening results (approx. 14.5 thousand per year - a figure estimated on the basis of data on the number of tests coming from the diagnostic phase) at the national level, the number of these patients may reach 900 per year.

The equipment of the facilities implementing the basic phase of the Programme should be considered as good. Elements necessary for proper collection of smears were provided by the vast majority of facilities. Similar equipment audit results were obtained for cytology clinics. On the other hand, some of the clinics carrying out the in-depth phase of the Programme are not equipped and prepared to perform colposcopy-targeted biopsies or to perform endocarvical curretage. These clinics should be eliminated from the Programme by the NHF's terminating the contract.

\section{Conclusions}

1. The way in which the facilities implementing the Cervical Cancer Prevention Programme are audited, dictated by the total lack of access to data collected by COK and auditors, requires fundamental changes. Developing an agreement between the Ministry of Health and the National Health Fund regarding access to data for the COK is essential for evaluation of results obtained within the Programme, calculation of quality measures and scientific evaluation of other aspects of the Programme.

2. Significanlty more Pap smears are collected within opportunistic screening as compared to the organised programme. Measures should be implemented to reduce opportunistic screening and to shift the stream of tests to the Programme. The great diversity of the ratio of tests performed within the Programme to tests performed outside the Programme by healthcare providers proves that shifting the implementation of screening procedures to the Programme is potentially possible.
3. The quality of smear sample collection, measured as a percentage of "unsuitable for evaluation" is the same for gynaecologists and midwives.

4. Access to the Programme should be increased through training and certification of midwives, who will be entitled to collect Pap smears both as part of the AOS and especially as part of the PCP, where only the Programme procedures are implemented, but the coverage of the Programme is very low.

5. External quality control of cervical screening tests should be implemented in laboratories and for individual cytotechnologists.

6. Measures should be taken to shift the implementation of diagnostic procedures in patients with positive test results to an in-depth phase in the Programme, which allows for the recording of data and the analysis of findings obtained through cytological, colposcopy and histopathological evaluations in terms of their quality.

7. The criteria of indications for histopathological verification for colposcopy at the in-depth phase should be unified and facilities where histopathological verification is not performed should be eliminated from the Programme.

8. Measures should be taken to reach approximately $6 \%$ of patients whose follow-up is unknown after obtaining a positive result of a cervical screening test in the Programme. There is no data on the follow-up of patients participating only in opportunistic screening, which is the predominant form of secondary prevention in Poland.

Conflict of interest: none declared

\section{Maryla Turkot, MSc}

Maria Skłodowska-Curie Memorial Cancer Centre

and Institute of Oncology

Department of Cancer Prevention

ul. Roentgena 5, 02-781 Warszawa, Poland

e-mail:maryla.turkot@coi.pl

Received: 3 Apr 2018

Accepted: 6 Jun 2018

\section{References}

1. Nowakowski A, Wojciechowska U, Wieszczy P et al. Trends in cervical cancer incidence and mortality in Poland: is there an impact of the introduction of the organised screening? Eur J Epidemiol 2017; 32: 529-532.

2. Arbyn M, Anttila A, Jordan J et al. (eds.). European guidelines for quality assurance in cervical cancer screening - Second edition. 2008. Luxembourg: Office to Official Publications of the European Communities, 2008.

3. Nowakowski A, Cybulski M, Sliwczynski A et al. The implementation of an organised cervical screening programme in Poland: an analysis of the adherence to European guidelines. BMC Cancer 2015; 15: 279. 


\section{Appendix}

Table I. Basic data on quality control of service providers implementing the basic phase of the Cervical Cancer Prevention Programme in 2016 and the first half of 2017

\begin{tabular}{|c|c|c|c|c|}
\hline Parameter & Min. & Max. & Mean & Median \\
\hline Number of the facility's working days per week & 3 & 6 & 5.3 & 6 \\
\hline Number of the facility's working hours per week & 2.5 & 69.5 & 28 & 25.8 \\
\hline Number of gynaecologists employed in the facility & 0 & 12 & 2.6 & 2 \\
\hline Number of midwives employed in the facility (collecting Pap smears) & 0 & 6 & 0.5 & 0 \\
\hline Number of days of waiting for the Pap smears collection, declared by providers & 0 & 90 & 5 & 1 \\
\hline Number of days of waiting for the Pap smears collection, verified by the auditors & 0 & 92 & 5.6 & 2.3 \\
\hline Number of Pap smears collected within the Programme for the whole 2016 & 0 & 1958 & 289 & 202.5 \\
\hline Number of Pap smears collected within the Programme in the first half of 2017 & 2 & 931 & 143.9 & 102 \\
\hline Number of Pap smears collected outside the Programme for the whole 2016 & 0 & 4214 & 550.1 & 334 \\
\hline Number of Pap smears collected outside the Programme in the first half of 2017 & 0 & 2141 & 298.4 & 182 \\
\hline $\begin{array}{l}\text { Ratio of the number of Pap smears collected within the Programme to those } \\
\text { collected outside the Programme in the whole audited period }\end{array}$ & 0 & 7.4 & 1 & 0.6 \\
\hline
\end{tabular}

Table II. Pap smears unsuitable for evaluation in the audited facilities of the basic phase of the Cervical Cancer Prevention Programme - all tests (within the Programme and outside it)

\begin{tabular}{lccc}
\hline Parameter & Min. & Max. & Mean \\
\hline Percentage of Pap smears unsuitable for evaluation in the audited facilities & 0 & 100 & 0.2 \\
$\begin{array}{l}\text { Percentage of Pap smears unsuitable for evaluation collected by } \\
\text { gynaecologists in the audited facilities }\end{array}$ & 0 & 33.7 & 0.9 \\
$\begin{array}{l}\text { Percentage of Pap smears unsuitable for evaluation collected by midwives in } \\
\text { the audited facilities }\end{array}$ & 0 & 100 & 0 \\
\hline
\end{tabular}

Table III. Follow-up of women with abnormal cervical screening test findings

\begin{tabular}{|c|c|c|c|}
\hline \multirow[t]{2}{*}{ Parameter } & \multicolumn{2}{|c|}{ Period, N (\%) } & \multirow[t]{2}{*}{ Total, N (\%) } \\
\hline & 01.01.-31.12.2016 & 01.01.-30.06.2017 & \\
\hline Positive results of Pap tests (ASC-US, LSIL, ASC-H, HSIL, AGC, cancer) & $1366(2.39)$ & $\begin{array}{l}656 \\
(2.30)\end{array}$ & $2022(2.36)$ \\
\hline $\begin{array}{l}\text { Women with further in-depth diagnosis or repeated Pap tests as } \\
\text { recommended by the SIMP }\end{array}$ & $\begin{array}{c}693 \\
(50.73)\end{array}$ & $\begin{array}{c}276 \\
(42.07)\end{array}$ & $\begin{array}{c}969 \\
(47.92)\end{array}$ \\
\hline $\begin{array}{l}\text { Women with neither further in-depth diagnosis nor Pap tests, as } \\
\text { recommended by the SIMP, but with reporting data from the National Health } \\
\text { Fund (NFZ) }\end{array}$ & $\begin{array}{l}115 \\
(8.42)\end{array}$ & $\begin{array}{c}53 \\
(8.08)\end{array}$ & $\begin{array}{c}168 \\
(8.31)\end{array}$ \\
\hline $\begin{array}{l}\text { Women for whom there is no in-depth diagnosis/repeated test data in the } \\
\text { SIMP or reporting data in the SIMP but there are data on patient follow-up/ } \\
\text { contacts in the facility documentation }\end{array}$ & $\begin{array}{c}195 \\
(14.28)\end{array}$ & $\begin{array}{c}143 \\
(21.80)\end{array}$ & $\begin{array}{c}338 \\
(16.72)\end{array}$ \\
\hline $\begin{array}{l}\text { Women for whom there is no in-depth diagnosis/repeated test data in the } \\
\text { SIMP or reporting data in the SIMP and there are not follow-up data in the } \\
\text { facility documentation }\end{array}$ & $\begin{array}{c}73 \\
(5.34)\end{array}$ & $\begin{array}{c}48 \\
(7.32)\end{array}$ & $\begin{array}{c}121 \\
(5.98)\end{array}$ \\
\hline
\end{tabular}


Table IV. Equipment in audited facilities implementing the basic stage of the Cervical Cancer Prevention Programme

\begin{tabular}{lcc}
\hline Piece of equipment & $\begin{array}{c}\text { Number of facilities having the } \\
\text { piece of equipment in question }\end{array}$ & $\begin{array}{c}\text { Percentage of facilities having the } \\
\text { piece of equipment in question }\end{array}$ \\
\hline Gynaecological chair & 196 & $99.0 \%$ \\
Disposable speculum & 197 & $99.5 \%$ \\
Disposable cytology brushes for simultaneous Pap smear collection & 197 & $99.5 \%$ \\
from the cervical orifice and the cervical canal) & 196 & $99.0 \%$ \\
Single-sided matte-finished microscopic slides & 195 & $98.5 \%$ \\
Cytofix (fixative) & 7 & $3.5 \%$ \\
Alcohol 96\% & 197 & $99.5 \%$ \\
Table for disposable gynaecological examination instruments & 197 & $99.5 \%$ \\
Shadowless lamp & 194 & $98.0 \%$ \\
Wash basin with elbow-operated basin mixer (soap dispenser and & & $99.5 \%$ \\
hand disinfectant dispenser) & 197 & $98.5 \%$ \\
Medical waste container & 195 & $97.0 \%$ \\
Computer with Internet access & 192 & $96.5 \%$ \\
Toilet seat & 191 & $97.5 \%$ \\
Bidet & 193 & \\
Wash basin & & \\
\hline
\end{tabular}

Table V. Figures for Pap tests performed in the periods under consideration

\begin{tabular}{llll}
\hline Parameter & $\begin{array}{l}\text { Number of Pap tests and } \\
\text { gynaecological examinations } \\
\text { performed within the Programme }\end{array}$ & $\begin{array}{l}\text { Number of Pap tests and } \\
\text { gynaecological examinations } \\
\text { performed outside the } \\
\text { Programme }\end{array}$ & $\begin{array}{l}\text { Number of all Pap tests: within the } \\
\text { Programme, outside the Programme, and } \\
\text { Pap tests other than gynaecological ones }\end{array}$ \\
\hline $\begin{array}{lll}\text { Period from } \\
01.01 .2016 \text { to }\end{array}$ & Min. 274 & Min. 232 & Min. 1104 \\
31.12 .2016 & Max. 49,598 & Max. 166,974 & Max. 185,728 \\
& Total: 544,352 & Total: $1,364,428$ & Total: $2,053,594$ \\
Median: 5264 & Median: 10,714 & Median: 15,925 \\
Mean: 8248 & Mean: 20,991 & Min. 603 \\
01.01 .2017 to & Min. 112 & Min. 103 & Max. 104,852 \\
30.06 .2017 & Max. 23,805 & Max. 95,617 & Total: 984,080 \\
& Total 257,288 & Total: 664,560 & Median: 8078 \\
& Median: 2430 & Median: 5314 & Mean: 15,140 \\
\hline
\end{tabular}

Table VI. Numbers and percentages of positive Pap test results diagnosed in audited cytology laboratories

\begin{tabular}{lll}
\hline Parameter & $\begin{array}{l}\text { Number of positive test results performed } \\
\text { within the Programme for all laboratories }\end{array}$ & $\begin{array}{l}\text { Percentage of positive test results performed } \\
\text { within the Programme for all laboratories }\end{array}$ \\
\hline Period from 01.01.2016 to 31.12.2016 & Min. 6 & Min. 0.52 \\
& Max. 1939 & Max. 9.32 \\
& Total: 14,589 & Median: 2.31 \\
& Median: 125 & Mean: 2.69 \\
& Mean: 221 & Min. 0.71 \\
Period from 01.01.2017 to 30.06 .2017 & Min. 1 & Max. 8.39 \\
& Max. 807 & Median: 2.31 \\
& Total: 6500 & Mean: 2.61 \\
\end{tabular}


Table VII. Numbers and percentages of particular categories of abnormal results of Bethesda system Pap tests diagnosed in the Programme in the audited laboratories in 2016

\begin{tabular}{lccccccccc}
\hline PARAMETER & \multicolumn{3}{c}{ Number } & \multicolumn{3}{c}{$\%$} \\
\cline { 2 - 11 } & Min. & Max. & Total & Median & Mean & Min. & Max. & Median & Mean \\
\hline ASC-US & 1 & 377 & 6054 & 52 & 91.73 & 0.11 & 5.14 & 0.90 & 1.21 \\
LSIL & 0 & 1457 & 5260 & 37 & 79.70 & 0.00 & 4.08 & 0.69 & 0.82 \\
ASC-H & 0 & 108 & 1169 & 8.5 & 17.71 & 0.00 & 0.73 & 0.19 & 0.21 \\
HSIL & 0 & 144 & 1508 & 12 & 22.85 & 0.00 & 1.73 & 0.26 & 0.30 \\
Squamous cell carcinoma & 0 & 8 & 97 & 1 & 1.47 & 0.00 & 0.13 & 0.01 & 0.02 \\
AGC & 0 & 61 & 479 & 3 & 7.26 & 0.00 & 2.37 & 0.06 & 0.13 \\
Adenocarcinoma in situ (AIS) & 0 & 5 & 5 & 0 & 0.08 & 0.00 & 0.01 & 0.00 & 0.00 \\
Adenocarcinoma & 0 & 3 & 4 & 0 & 0.06 & 0.00 & 0.02 & 0.00 & 0.00 \\
Other cancers & 0 & 2 & 4 & 0 & 0.06 & 0.00 & 0.05 & 0.00 & 0.00 \\
Pap smear samples unsuitable for & 0 & 450 & 3196 & 11 & 48.42 & 0 & 3.08 & 0.31 & 0.56 \\
evaluation & & & & & & & & & \\
\hline
\end{tabular}

Table VIII. Numbers and percentages of particular categories of abnormal results of Bethesda system Pap tests diagnosed in the Programme in the audited laboratories in the first half of 2017

\begin{tabular}{|c|c|c|c|c|c|c|c|c|c|}
\hline \multirow[t]{2}{*}{ PARAMETER } & \multicolumn{5}{|c|}{ Number } & \multicolumn{4}{|c|}{$\%$} \\
\hline & Min. & Max. & Total & Median & Mean & Min. & Max. & Median & Mean \\
\hline ASC-US & 0 & 175 & 2679 & 21 & 40.60 & 0.00 & 5.27 & 0.86 & 1.18 \\
\hline LSIL & 0 & 606 & 2293 & 18 & 34.74 & 0.00 & 3.32 & 0.66 & 0.76 \\
\hline ASC-H & 0 & 47 & 601 & 5 & 9.12 & 0.00 & 0.65 & 0.18 & 0.23 \\
\hline HSIL & 0 & 77 & 696 & 5.5 & 10.55 & 0.00 & 1.90 & 0.27 & 0.32 \\
\hline Squamous cell carcinoma & 0 & 4 & 42 & 0 & 0.64 & 0.00 & 0.24 & 0.00 & 0.03 \\
\hline AGC & 0 & 20 & 183 & 1 & 2.78 & 0.00 & 1.79 & 0.05 & 0.10 \\
\hline Adenocarcinoma in situ (AIS) & 0 & 5 & 6 & 0 & 0.09 & 0.00 & 0.03 & 0.00 & 0.00 \\
\hline Adenocarcinoma & 0 & 1 & 3 & 0 & 0.05 & 0.00 & 0.03 & 0.00 & 0.00 \\
\hline Other cancers & 0 & 1 & 1 & 0 & 0.02 & 0.00 & 0.01 & 0.00 & 0.00 \\
\hline $\begin{array}{l}\text { Pap smear samples unsuitable for } \\
\text { evaluation }\end{array}$ & 0 & 218 & 1536 & 6 & 23.27 & 0 & 8.82 & 0.28 & 0.65 \\
\hline
\end{tabular}

Table IX. Basic data on discrepancies between the primary and secondary evaluation of Pap smear samples found during the internal quality control of the evaluation of Pap smear samples in the audited laboratories

\begin{tabular}{lll}
\hline Parameter & $\begin{array}{l}\text { Percentage of tests re-evaluated in quality } \\
\text { control procedures }\end{array}$ & $\begin{array}{l}\text { Percentage of discrepancies detected } \\
\text { between primary and secondary } \\
\text { evaluation of samples in quality control } \\
\text { procedures }\end{array}$ \\
\hline Period from 01.01 .2016 to 31.12 .2016 & Min. 7.2 & Min. 0 \\
Max. 100 & Median. 10.5 & Median. 0.3 \\
Mean. 23.1 & Mean. 1.5 \\
Period from 01.01 .2017 to 30.06 .2017 & Min. 6.9 & Min. 0 \\
& Max. 100 & Max. 12.8 \\
Median. 11.5 & Median. 0.3 \\
\hline
\end{tabular}


Table X. The general evaluation of the audit of cytological laboratories implementing the diagnostic phase of the Cervical Cancer Prevention Programme and the data concerning the fulfilment of particular conditions of the Programme implementation

\begin{tabular}{|c|c|}
\hline \multicolumn{2}{|c|}{$\begin{array}{l}\text { Criteria for the provision of services guaranteed by the Cervical Cancer Prevention Programme - diagnostic phase in accordance with } \\
\text { the regulation of the Minister of Health dated August 5, } 2016 \text { on detailed criteria for selection of offers in the procedure for conclusion of } \\
\text { agreements on provision of healthcare services and the Regulation of the Minister of Health of } 2013 \text { on guaranteed services within the scope } \\
\text { of health programmes }\end{array}$} \\
\hline Criterion & $\begin{array}{l}\text { Number and percentage of facilities } \\
\text { meeting this criterion }\end{array}$ \\
\hline $\begin{array}{l}\text { Medical diagnostic laboratory entered in the register kept by the National Council of Laboratory } \\
\text { Diagnostics or a pathomorphological facility with a cytological lab }\end{array}$ & $66(100 \%)$ \\
\hline A doctor specializing in pathomorphology or pathological anatomy & $66(100 \%)$ \\
\hline $\begin{array}{l}\text { Lab cytotechnologists with a title of specialist in medical cytomorphology or a proven ability and } \\
\text { experience relevant to the performance of cytological tests }\end{array}$ & $65(100 \%)-1$ centre, no data available \\
\hline $\begin{array}{l}\text { Medical equipment and instruments: high-quality microscopes capable of giving a magnification of at } \\
\text { least } 400 \text { times }\end{array}$ & $66(100 \%)$ \\
\hline $\begin{array}{l}\text { Performed by each person* evaluating the samples in at least } 7000 \text { Pap tests a year } \\
\text { * qualifications of the staff in accordance with the regulation (the number of tests carried out refers to the } \\
\text { whole operations, also outside the contract with the National Health Fund) }\end{array}$ & $\begin{array}{l}47(73.4 \%)-2 \text { centres, no data } \\
\text { available }\end{array}$ \\
\hline $\begin{array}{l}\text { Performing } 15,000 \text { Pap tests at the laboratory** } \\
* * \text { refers to the whole operations, also outside the contract with the National Health Fund) }\end{array}$ & $45(68.2 \%)$ \\
\hline $\begin{array}{l}\text { Sending the result to the clinic that collected the test material within seven working days from the date } \\
\text { of the sample reception }\end{array}$ & $\begin{array}{l}63(96.9 \%)-1 \text { centre, no data } \\
\text { available }\end{array}$ \\
\hline \multicolumn{2}{|c|}{$\begin{array}{l}\text { Detailed, supplementary conditions concerning the services provision terms in accordance with the order no. 99/2015/DSOZ of the President } \\
\text { of the National Health Fund of } 22 \text { December } 2015 \text { - requirements for tenderer at the diagnostic phase }\end{array}$} \\
\hline Condition & $\begin{array}{l}\text { Number and percentage of facilities } \\
\text { meeting this criterion }\end{array}$ \\
\hline $\begin{array}{l}\text { A cytological sample other than with a negative result must be evaluated in two steps: at least by a } \\
\text { doctor specialising in pathomorphology or pathological anatomy }\end{array}$ & $66(100 \%)$ \\
\hline
\end{tabular}

Table XI. Basic data on the working time of facilities and the number of employees at healthcare providers executing the phase of in-depth diagnostics of the Cervical Cancer Prevention Programme in 2016 and the first half of 2017

\begin{tabular}{lcccc}
\hline Parameter & Min. & Max. & Mean & Median \\
\hline Number of the facility's working days per week & 1 & 6 & 3.5 & 4 \\
Number of the facility's working hours per week & 1 & 60 & 21.5 & 12 \\
Number of gynaecologists employed in the facility (2016) & 1 & 7 & 7.8 & 1.5 \\
Number of gynaecologists employed in the facility (2017) & 1 & 30 & 7.3 & 7 \\
$\begin{array}{l}\text { Number of days of waiting for colposcopy, declared by healthcare } \\
\text { providers }\end{array}$ & 0 & 25 & 8.3 & 6.8 \\
Number of days of waiting for colposcopy, verified by auditors & 0 & &
\end{tabular}


Table XII. Number of colposcopies performed in the laboratories during the audited periods

\begin{tabular}{ll}
\hline 01.01.2016-31.12.2016* & \\
\hline Within the Programme & Outside the Programme \\
Number: & Number: \\
Min. 0 & Min. 0 \\
Max. 573 & Mean: 1535.8 \\
Mean: 46.2 & Median: 38.5 \\
Median: 22.5 & Total for all facilities: 9037 \\
Total for all facilities: 2740 & \\
\hline 01.01.2017-30.06.2017* & \\
\hline Within the Programme & Outside the Programme \\
Number: & Number: \\
Min. 1 & Min. 0 \\
Max. 220 & Max. 684 \\
Mean: 20.3 & Mean: 79.3 \\
Median: 10 & Median: 25 \\
Total for all facilities: 1189 & Total for all facilities 4599 \\
\hline
\end{tabular}

*4 laboratories did not provide any data on the number of tests performed outside the Programme

Table XIII. Numbers of biopsies performed in the laboratories and their percentage in relation to the number of colposcopies performed in the audited periods

\begin{tabular}{ll}
\hline 01.01.2016-31.12.2016* & \\
\hline Number of cervical biopsies & Percentage of biopsies in relation to the number of colposcopies \\
Number: & Min. $0 \%$ \\
Min. 0 & Max. $100 \%$ \\
Max. 547 & Mean: $30.4 \%$ \\
Mean: 36.1 & Median: $16.3 \%$ \\
Median: 17 & \\
Total for all facilities: 2236 & \\
\hline $01.01 .2017-30.06 .2017^{*}$ & Percentage of biopsies in relation to the number of colposcopies \\
\hline Number of cervical biopsies & Number: \\
Number: & Min. $0 \%$ \\
Min. 0 & Max. $100 \%$ \\
Max. 207 & Mean: $28.4 \%$ \\
Mean: 15.5 & Median: $15.4 \%$ \\
Median: 6.5 & \\
Total for all facilities: 962 & \\
\hline
\end{tabular}

*4 laboratories did not provide any data on the number of tests performed outside the Programme 
Table XIV. Equipment in audited facilities implementing the in-depth phase of the Cervical Cancer Prevention Programme

\begin{tabular}{|c|c|c|}
\hline Piece of equipment & $\begin{array}{l}\text { Number of facilities having the piece } \\
\text { of equipment in question }\end{array}$ & $\begin{array}{l}\text { Percentage of facilities having the } \\
\text { piece of equipment in question }\end{array}$ \\
\hline Optical colposcope with a valid technical passport & 62 & $100 \%$ \\
\hline Contrasting filter in a colposcope & 59 & $95.2 \%$ \\
\hline Disposable specula & 62 & $100 \%$ \\
\hline Sterilised reusable specula & 29 & $46.8 \%$ \\
\hline Tweezers & 61 & $98.4 \%$ \\
\hline Biopsy forceps for colposcopy-targeted biopsy & 59 & $95.2 \%$ \\
\hline Cervical scrapers & 58 & $93.6 \%$ \\
\hline Buffered formalin & 59 & $95.2 \%$ \\
\hline Containers for tissue material fixation & 61 & $98.4 \%$ \\
\hline Sterile swabs & 62 & $100 \%$ \\
\hline Physiological saline solution & 62 & $100 \%$ \\
\hline Acetic acid 3-5\% & 62 & $100 \%$ \\
\hline Lugol's iodine & 58 & $93.6 \%$ \\
\hline $\begin{array}{l}\text { Electrocautery apparatus and ball coagulation electrodes for post- } \\
\text { biopsy haemostasis }\end{array}$ & 49 & $79.0 \%$ \\
\hline Coagulating laser & 17 & $27.4 \%$ \\
\hline Autoclave & 48 & $77.4 \%$ \\
\hline Separate sterilisation room & 50 & $80.7 \%$ \\
\hline Sterilisation procedure in place & 52 & $83.9 \%$ \\
\hline External sterilisation outside the facility & 11 & $17.7 \%$ \\
\hline Use of disposable instruments only & 6 & $9.7 \%$ \\
\hline Availability of HPV diagnostics & 42 & $67.7 \%$ \\
\hline
\end{tabular}

